\title{
Far-fields Radiated of a Small Circular Loop Antenna Utilized in Remote Probing of the Earth
}

\author{
Hanan Shehata Shoeib \\ Department of Basic Sciences, College of Education, Imam Abdulrahman Bin Faisal University, Dammam, Saudi Arabia
}

Email address:

hanan.shehata@yahoo.com, hashoeib@iau.edu.sa

\section{To cite this article:}

Hanan Shehata Shoeib. Far-fields Radiated of a Small Circular Loop Antenna Utilized in Remote Probing of the Earth. American Journal of Applied Mathematics. Vol. 7, No. 4, 2019, pp. 90-97. doi: 10.11648/j.ajam.20190704.11

Received: July 14, 2019; Accepted: August 27, 2019; Published: September 9, 2019

\begin{abstract}
In a recent study, the classical problem of a circular loop antenna carrying a uniform current on the Earth's surface has been revisited, with a scope for deriving Closed-form formulae for the generated magnetic and electric far fields by a vertical magnetic dipole (VMD) located at certain height above the surface of a planar two-layer conducting earth, with a high degree of accuracy. The solution is obtained by reducing the field integrals to combinations of known Sommerfeld integrals (SIs), which is advantageous over the previous numerical and analytical-numerical approaches, and its usage takes negligible computation time. Numerical simulations are performed and illustrated by figures for different values of the frequency to show the accuracy of the obtained field expressions and to investigate the behavior of the above surface ground fields in a wide frequency range. Results can be used to evaluate numerical solutions of more complicated modeling algorithms, for application to mobile communication and will be useful for remote sensing especially when the transmitter is close to the surface.
\end{abstract}

Keywords: Far-field, Vertical Magnetic Dipole (VMD) Radiation, Circular Loop Antenna, Planar Layered Conducting Earth, Sommerfeld Integral (SI)

\section{Introduction}

The need to evaluate the electromagnetic fields radiated by horizontal or vertical loop antennas located in layered media arises in a variety of applications of scientific interest, especially in the areas of electromagnetics [1,2], close-to the-surface radio communication $[3,4]$, remote sensing and geophysical prospecting [4-7].

In most cases, these fields may be expressed in terms of Sommerfeld-type integrals, which are highly oscillatory in nature, with poor convergence properties. This makes numerical integration difficult and impractical, especially for the surface-to surface propagation case and rather difficult to evaluate. So, many approaches have been proposed for accurately evaluating the integral representations describing the generated electromagnetic field (EMF) components.

Sommerfeld integrals (SIs) can be evaluated with numerical integration routines, but it becomes very tedious when the observation point is in the far-field of source, because the integrand is rapidly oscillating. Fortunately, in the far-field region, we can use either the stationary phase method or the saddle-point method to evaluate the integral to obtain a far-field expression [1, 5, 8-11], but these methods involve lengthy algebra and several transformations. As an alternative, Chew [12] presented a method that captures the essence of the stationary phase method and derived the farfield approximations of the integral in a few easily remembered steps. Lately, Long et al. [13] developed Chew technique and with the help of the complex image theory [14], he derived expression of the SI rapidly for the far-field depending on the stationary phase-point. Recently, many researchers such as Parise et al. [15] studied the problem of a large circular loop antenna carrying uniform current and situated at the Earth's surface. They obtained a procedure that allows to derive the exact canonical solution to the problem. The canonical solution describes all the radiated field components, and is valid in both the quasi-static and nonquasi-static frequency regions, also the computational cost of the canonical solution is negligible with respect to that of numerical techniques commonly used to evaluate Sommerfeld-type integrals.

The present work focuses on the derivation of Closed-form formulae for Electromagnetic far-fields due to a VMD (horizontal circular loop antenna) carrying uniform current 
and lying at a certain height (h) in air on a planar two-layered conducting earth. To derive the solutions, firstly we began with the wave equation of the scalar magnetic Hertz potential $\Pi$ to find the electric and magnetic fields as Sl representation. Secondly, by the technique [13], we evaluated those integrals. The integration was divided into two factors; one is the slow factor, while the other one is the rapid factor, and by using the complex image theory [14] the reflection coefficient was replaced by an exponential function which tends to the summation of algebraic series. Then, the closedform expressions for the far-field in the first region of the medium due to a vertical magnetic dipole in the air obtained rapidly.

The computation method takes into consideration the effects of both conduction as well as displacement currents, and is well suitable for any position of the source loop either in the air or on the surface of the model, in contrary to the earlier methods which face convergence problem. The physical meaning of the relationships between the vertical magnetic field (VMF) and its frequencies were represented as a summation of a series of waves which propagate upward from the source and another series of waves which propagate firstly downward from the source then reflect upward at the bottom of the overburden slab, and travel along the same path as the former mentioned waves. The results agree with those results obtained by using the conventional stationary phase method or saddle-point method but it is much easier and more quickly. This method can be easily used to calculate the far-fields for other similar problems.

\section{Planar Earth Model and Formulation}

The geometry of the VMD (circular loop antenna) and the electromagnetic parameters of the planar two-layer conducting earth are illustrated in Figure 1.

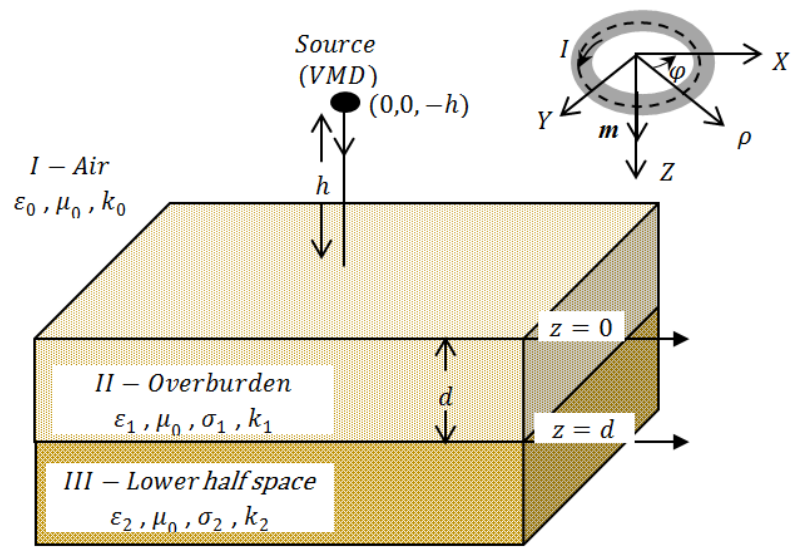

Figure 1. Layered earth model and measurement system under consideration.

The emitter lies at a fixed height (h) above the earth's surface, has area (dA), and carries a current equal to $I$. The air-earth interface is at $z=0$ in Cartesian coordinate system $(x, y, z)$. The earth's adopted model consists of a homogeneous overburden slab of thickness $(d)$ above the half-space. $\varepsilon_{i}, \mu_{i}, \sigma_{i}, k_{i}$ denote permittivity, permeability, conductivity, and propagation constant, where $i=0,1,2$ denotes air $(-h \leq z \leq 0)$, overburden slab $(0 \leq z \leq d)$, and lower half space $(d \leq z \leq \infty)$, respectively. The magnetic permeability is taken to be equal to that of free space throughout.

It is well known that the electromagnetic field generated by a vertical magnetic dipole can be derived from the magnetic Hertz vector $\vec{\Pi}(r)$ at an observation point $P(\rho, z)$ in cylindrical coordinate system $(\rho, \varphi, z)$ in the air region, due to the source, of which only one component $\Pi_{z}(\vec{r})$ in the $z$ direction is different from zero [16]. The electric field vector $\vec{E}(\vec{r})$ and the magnetic field vector $\vec{H}(\vec{r})$ are expressed in terms of $\vec{\Pi}(\vec{r})$ by the following formulas:

$$
\vec{E}(\vec{r})=-i \omega \mu_{0} \nabla \times \vec{\Pi}_{m}(\vec{r}), \vec{H}(\vec{r})=\nabla \times \nabla \times \vec{\Pi}_{m}(\vec{r})
$$

The magnetic Hertz vector has only a $z$-component $\Pi_{z}$, and it satisfies the Helmholtz wave equation for a time factor $e^{i \omega t}$ (where $i=\sqrt{-1}$ and $\omega$ is the angular frequency of the driving source), as is well known. The fields components are given by the following formulas [17]:

$$
E_{\varphi}(\rho, z)=i \omega \mu_{0} \frac{\partial \Pi_{z}}{\partial \rho}, H_{\rho}=\frac{\partial^{2} \Pi_{z}}{\partial \rho \partial z}, H_{z}=-\left(\frac{\partial}{\partial \rho^{2}}+\frac{1}{\rho} \frac{\partial}{\partial \rho}\right) \Pi_{z}
$$

The tangential components of either the electric or magnetic field strength must be continuous through the boundary surface of the two media, so the Hertz vector and its derivative are continuous [10]. Then

$$
\begin{aligned}
& \Pi_{z o}=\Pi_{z 1}, \frac{\partial \Pi_{z 0}}{\partial z}=\frac{\partial \Pi_{z 1}}{\partial z} \text { at } \mathrm{z}=0 \\
& \Pi_{z 1}=\Pi_{z 2}, \frac{\partial \Pi_{z 1}}{\partial z}=\frac{\partial \Pi_{z 2}}{\partial z} \text { at } \mathrm{z}=\mathrm{d}
\end{aligned}
$$

A further condition that must be also satisfied is the condition of finiteness of the field at the infinity (Sommerfeld radiation condition).

\section{The Integral Representation of the Field}

According to Sommerfeld [11], the primary and secondary field at the general observation point $P(r, z)$ take the form

$$
\begin{aligned}
& \Pi_{P}(\rho, z)=\frac{I d A}{8 \pi} \int_{-\infty}^{\infty} \frac{\lambda}{u} e^{-u|z+h|} H_{0}^{(2)}(\lambda \rho) d \lambda \\
& \Pi_{S}(\rho, z)=\frac{I d A}{8 \pi} \int_{-\infty}^{\infty}\left\{\begin{array}{c}
f_{i}(\lambda) e^{u(z-h)}+ \\
f_{j}(\lambda) e^{-u(z-h)}
\end{array}\right\} H_{0}^{(2)}(\lambda \rho) d \lambda
\end{aligned}
$$

Where $u=\sqrt{\lambda^{2}-k^{2}}$ (the root with positive real part must be taken), $H_{0}^{(2)}(\lambda \rho)$ is the Zeroth-order Hankel function of the second kind, and $f_{i j}$ are constant functions which can be determined easily from the boundary conditions.

The non-vanishing components of the electric and magnetic fields $E_{\varphi}, H_{\rho}, H_{z}$ in the three regions can be deduced without much labor by substituting from equations (2) in equations (5), (6) as follows, considering that the regions I and III are infinite upward and downward, 
respectively, along z-axis:

In I-region (air) $-\infty \leq \mathrm{z} \leq 0$ :

$$
\begin{aligned}
& E_{\varphi o}(\rho, z)=\frac{-i \omega \mu_{0} I d A}{8 \pi} \int_{-\infty}^{\infty}\left[\frac{\lambda}{u_{0}} e^{-u_{0}(z+h)}+f_{0}(\lambda) e^{u_{0}(z-h)}\right] \lambda H_{1}^{(2)}(\lambda \rho) d \lambda \\
& H_{\rho o}(\rho, z)=\frac{-I d A}{8 \pi} \int_{-\infty}^{\infty}\left[\frac{\lambda}{u_{0}} e^{-u_{0}(z+h)}+f_{0}(\lambda) e^{u_{0}(z-h)}\right] \lambda u_{0} H_{1}^{(2)}(\lambda \rho) d \lambda \\
& H_{z o}(\rho, z)=\frac{I d A}{8 \pi} \int_{-\infty}^{\infty}\left[\frac{\lambda}{u_{0}} e^{-u_{0}(z+h)}+f_{0}(\lambda) e^{u_{0}(z-h)}\right] \lambda^{2} H_{0}^{(2)}(\lambda \rho) d \lambda
\end{aligned}
$$

In II- region (overburden) $0 \leq \mathrm{z} \leq d$ :

$$
\begin{aligned}
& E_{\varphi 1}(\rho, z)=\frac{-i \omega \mu_{0} I d A}{8 \pi} \int_{-\infty}^{\infty}\left[\frac{\lambda}{u_{1}} e^{u_{1}(z-h)}+f_{1}(\lambda) e^{-u_{1}(z-h)}+f_{2}(\lambda) e^{u_{1}(z-h)}\right] \lambda H_{1}^{(2)}(\lambda \rho) d \lambda \\
& H_{\rho 1}(\rho, z)=\frac{-I d A}{8 \pi} \int_{-\infty}^{\infty}\left[\frac{-\lambda}{u_{1}} e^{u_{1}(z-h)}-f_{1}(\lambda) e^{-u_{1}(z-h)}+f_{2}(\lambda) e^{u_{1}(z-h)}\right] \lambda u_{1} H_{1}^{(2)}(\lambda \rho) d \lambda \\
& H_{z 1}(\rho, z)=\frac{I d A}{8 \pi} \int_{-\infty}^{\infty}\left[\frac{\lambda}{u_{1}} e^{u_{1}(z-h)}+f_{1}(\lambda) e^{-u_{1}(z-h)}+f_{2}(\lambda) e^{u_{1}(z-h)}\right] \lambda^{2} H_{0}^{(2)}(\lambda \rho) d \lambda
\end{aligned}
$$

In III- region (lower half space) $d \leq \mathrm{z} \leq \infty$ :

$$
\begin{aligned}
& E_{\varphi 2}(\rho, z)=\frac{-i \omega \mu_{0} I d A}{8 \pi} \int_{-\infty}^{\infty} f_{3}(\lambda) e^{-u_{2}(z-h)} \lambda H_{1}^{(2)}(\lambda \rho) d \lambda \\
& H_{\rho 2}(\rho, z)=\frac{I d A}{8 \pi} \int_{-\infty}^{\infty} f_{3}(\lambda) e^{-u_{2}(z-h)} \lambda u_{2} H_{1}^{(2)}(\lambda \rho) d \lambda \\
& H_{z 2}(\rho, z)=\frac{I d A}{8 \pi} \int_{-\infty}^{\infty} f_{3}(\lambda) e^{-u_{2}(z-h)} \lambda^{2} H_{0}^{(2)}(\lambda \rho) d \lambda
\end{aligned}
$$

where $H_{1}^{(2)}(\lambda \rho)$ is the first-order Hankel function of the second kind, and $H_{n}^{(1)}(-x)=H_{2}^{(2)}(x)$ (half - circuit relation [18]).

From the boundary conditions (3), (4) and by using purely algebraic process, we can find explicitly the unknown functions $f_{i}(\lambda)$ as follows:

$$
\begin{gathered}
f_{0}(\lambda)=\frac{\lambda}{u_{0}}\left[\frac{r_{01}+r_{12} e^{-2 u_{1} d}}{1+r_{01} r_{12} e^{-2 u_{1} d}}\right], f_{1}(\lambda)=\frac{\left(\frac{2 \lambda}{\mathrm{u}_{01}^{+}}\right) e^{-\mathrm{u}_{01}^{+} h}}{1+r_{01} r_{12} e^{-2 u_{1} d}} \\
f_{2}(\lambda)=\frac{\left(\frac{2 \lambda}{\mathrm{u}_{01}^{+}}\right) r_{12} e^{-\mathrm{u}_{01}^{-} h-2 u_{1} d}-\left(\frac{\lambda}{u_{1}}\right)\left(1+r_{01} r_{12} e^{-2 u_{1} d}\right)}{1+r_{01} r_{12} e^{-2 u_{1} d}}, f_{3}(\lambda)=\frac{\left(\frac{4 u_{1} \lambda}{\mathrm{u}_{01}^{+} \mathrm{u}_{12}^{+}}\right) e^{-\mathrm{u}_{12}^{-} d-\mathrm{u}_{12}^{+} h}}{1+r_{01} r_{12} e^{-2 u_{1} d}}
\end{gathered}
$$

where

$$
r_{01}=\frac{u_{01}^{-}}{u_{01}^{+}}=\frac{u_{0}-u_{1}}{u_{0}+u_{1}}, r_{12}=\frac{u_{12}^{-}}{u_{12}^{+}}=\frac{u_{1}-u_{2}}{u_{1}+u_{2}}
$$

where $r_{01}$ and $r_{12}$ are Fresnel reflection coefficients at the interfaces $\mathrm{z}=0, \mathrm{~d}$, respectively.

Paying attention to equations (7)-(15), we are sure that the term of the integral containing $e^{u_{i}(z+h)}$ is the direct (primary) wave from the source to the observing point. This term is important, if the transmitter and receiver are set very closely. The other terms are the secondary wave which is consisting of two waves, one is incident (downward) containing $e^{u_{0}(z-h)}$ with the positive $\mathrm{z}$ direction and the other is reflected (upward) containing $e^{-u_{0}(z-h)}$ with the negative $z$-direction. The terms containing $e^{-u_{0}(z-h)}$ in first-region and $e^{u_{2}(z-h)}$ in thirdregion are not permitted since they would violate the Sommerfeld radiation condition [11] at $|z| \rightarrow \infty$.

\section{Estimate of the Integral in the Far-Field}

The scope of this work is to exactly evaluate the field components in the far field at the air. To this goal, we focus our attention on (9) to estimate $H_{z o}$ and the other components $E_{\varphi o}$ and $H_{\rho o}$ can also estimate in similar way. The magnetic field on the first boundary where $\mathrm{z}=0$, could be found using (9) and (16) as follows

$$
H_{z o}(\rho, 0)=\frac{I d A}{8 \pi} \int_{-\infty}^{\infty}\left[\frac{\lambda^{3}}{u_{0}} e^{-u_{0} h}\left(1+R_{0}\right) H_{0}^{(2)}(\lambda \rho) d \lambda\right.
$$

where

$$
R_{0}(\lambda)=\frac{r_{01}+r_{12} e^{-2 u_{1} d}}{1+r_{01} r_{12} e^{-2 u_{1} d}}
$$

Substituting from (18) in (19) and by using easy algebraic process, we have

$$
H_{z o}(\rho, 0)=\frac{I d A}{8 \pi} \int_{-\infty}^{\infty}\left[\frac{\lambda^{3}}{u_{0}} e^{-u_{0} h}\left[\frac{\left(1-r_{10}\right)\left(1+r_{12} e^{-2 u_{1} d}\right)}{1-r_{10} r_{12} e^{-2 u_{1} d}}\right] H_{0}^{(2)}(\lambda \rho) d \lambda\right.
$$


where $r_{01}=-r_{10}$.

Equation (20) can be written in another form as follows

$$
H_{z o}(\rho, 0)=\frac{I d A}{8 \pi} \int_{-\infty}^{\infty}\left[\frac{\lambda^{3}}{u_{0}} e^{-u_{0} h}\left[\frac{\left(1-\frac{u_{10}^{-}}{u_{10}^{+}}\right)\left(1+\frac{u_{12}^{-}}{u_{12}^{+}} e^{-2 u_{1} d}\right)}{1-\frac{u_{10}^{-}}{u_{10}^{+}} \frac{u}{u_{12}^{+}} e^{-2 u_{1} d}}\right] H_{0}^{(2)}(\lambda \rho) d \lambda\right.
$$

Let us consider $X=r_{10} r_{12} e^{-2 u_{1} d}=\frac{u_{10}^{-}}{u_{10}^{+}} \frac{u_{12}^{-}}{u_{12}^{+}} e^{-2 u_{1} d}$, and noting that $\left|\frac{u_{10}^{-}}{u_{10}^{+}} \frac{u_{12}^{-}}{u_{12}^{+}} e^{-2 u_{1} d}\right|<1, \frac{1}{1-\mathrm{X}}=\sum_{0}^{\infty} x^{m}$.

So the denominator in (13) can be expanded as an infinite series and $H_{z o}$ turns into

$$
H_{z o}(\rho, 0)=\frac{I d A}{8 \pi} \int_{-\infty}^{\infty}\left[\frac{\lambda^{3}}{u_{0}} e^{-u_{0} h}\left(1-\frac{u_{10}^{-}}{u_{10}^{+}}\right)\left(1+\frac{u_{12}^{-}}{u_{12}^{+}} e^{-2 u_{1} d}\right)\left[\sum_{0}^{\infty}\left(\frac{u_{10}^{-}}{u_{10}^{+}} \frac{u_{12}^{-}}{u_{12}^{+}} e^{-2 u_{1} d}\right)^{m}\right] H_{0}^{(2)}(\lambda \rho) d \lambda\right.
$$

According to the complex image theory [14], when the condition $\left|k_{1}^{2}\right| \gg\left|k_{0}^{2}\right|$ is satisfied, we have

$$
\frac{u_{10}^{-}}{u_{10}^{+}} \approx e^{-u_{0} a} \frac{2 u_{0}}{u_{10}^{+}} \approx 1-e^{-u_{0} a}
$$

where $a=\frac{-2 i}{u_{1}}$

For the case we discuss, there is always $\left|k_{1}^{2}\right| \gg 80\left|k_{0}^{2}\right|$ for any frequency [10]. Under these conditions and using the first formula of (23), equation (22) can be written as

$$
H_{z o}(\rho, 0)=\frac{I d A}{8 \pi}\left(\sum_{m=0}^{\infty}[\Phi(m)+\Psi(m)+\beta(m)+\gamma(m)]\right)
$$

where

$$
\begin{gathered}
\Phi(m)=\int_{-\infty}^{\infty} \frac{\lambda^{3}}{u_{0}}\left(\frac{u_{12}^{-}}{u_{12}^{+}}\right)^{m} e^{-u_{0}(m a+h)-2 u_{1} m d} H_{0}^{(2)}(\lambda \rho) \lambda d \lambda \\
\Psi(m)=\int_{-\infty}^{\infty} \frac{\lambda^{3}}{u_{0}}\left(\frac{u_{12}^{-}}{u_{12}^{+}}\right)^{m+1} e^{-u_{0}(m a+h)-2 u_{1}(m+1) d} H_{0}^{(2)}(\lambda \rho) \lambda d \lambda \\
\beta(m)=\int_{-\infty}^{\infty} \frac{\lambda^{3}}{u_{0}}\left(\frac{u_{12}^{-}}{u_{12}^{+}}\right)^{m} e^{-u_{0}[(m+1) a+h]-2 u_{1} m d} H_{0}^{(2)}(\lambda \rho) \lambda d \lambda \\
\gamma(m)=\int_{-\infty}^{\infty} \frac{\lambda^{3}}{u_{0}}\left(\frac{u_{12}^{-}}{u_{12}^{+}}\right)^{m+1} e^{-u_{0}[(m+1) a+h]-2 u_{1}(m+1) d} H_{0}^{(2)}(\lambda \rho) \lambda d \lambda
\end{gathered}
$$

First, we estimated $\Phi(m)$, which can be arranged properly as

$$
\Phi(m)=\int_{-\infty}^{\infty}\left[\lambda^{2}\left(\frac{u_{12}^{-}}{u_{12}^{+}}\right)^{m} e^{-2 u_{1} m d}\right]\left[\frac{\lambda}{u_{0}} e^{-u_{0}(m a+h)} H_{0}^{(2)}(\lambda \rho)\right] d \lambda
$$

When $\rho \rightarrow \infty$, the first factor of (18) is slowly varying while the second one is rapidly varying. Using the simple technique [13], then equation (29) can be written as

$$
\Phi(m)=\int_{-\infty}^{\infty} f(\lambda) h(\lambda, \rho) d \lambda
$$

Comparing both equations (29) and (30) we find that the rapidly varying part:

$$
h(\lambda, \rho) \approx e^{i \rho S(\lambda)} \approx \frac{\lambda}{u_{0}} e^{-u_{0}(m a+h)} H_{0}^{(2)}(\lambda \rho), \rho \rightarrow \infty
$$

In fact, when $\rho \rightarrow \infty$ and for large values of $|\lambda|$ the Hankel function can be replaced with its asymptotic form [18]

$$
H_{n}^{(2)}(\lambda \rho) \approx \sqrt{\frac{2}{\pi \lambda \rho}} e^{i\left(\lambda \rho-\frac{n \pi}{2}-\frac{\pi}{4}\right)}
$$

Substituting from (32) in (31) we get:

$$
e^{i \rho S(\lambda)} \approx \frac{\lambda}{u_{0}} e^{-u_{0}(m a+h)} \sqrt{\frac{2}{\pi \lambda \rho}} e^{i\left(\lambda \rho-\frac{\pi}{4}\right)}
$$

By comparing both side of (33) we can find that: 


$$
S(\lambda) \approx-i \lambda \rho-i(m a+h) \sqrt{k_{0}^{2}-\lambda^{2}}, u_{0}=i \sqrt{k_{0}^{2}-\lambda^{2}}
$$

The location of the stationary phase point $\lambda_{0}$ of $h(\lambda, \rho)$ is given by $S^{\prime}\left(\lambda_{0}\right)=0$, then

$$
\left.\frac{\partial}{\partial \lambda} i\left[-\lambda \rho-(m a+h) \sqrt{k_{0}^{2}-\lambda^{2}}\right]\right|_{\lambda=\lambda_{0}}=0
$$

The solution of (34) is:

$$
\lambda_{0}=\frac{\rho k_{0}}{r_{1}}, \text { with } r_{m}=\sqrt{\rho^{2}+(m a+h)^{2}}
$$

Most of the contribution to the integral (30) is from the vicinity of the point $\lambda=\lambda_{0}$, if $f(\lambda)$ has no singularities in the vicinity of $\lambda_{0}$, then equation (30) becomes

$$
\Phi(m)=f\left(\alpha_{0}\right) \int_{-\infty}^{\infty} h(\lambda, \rho) d \lambda
$$

Using the well-known Sommerfeld integral:

$$
\frac{e^{-i k r}}{r}=\frac{1}{2} \int_{-\infty}^{\infty} \frac{\lambda}{u} e^{-\mathrm{u}|z|} H_{0}^{(2)}(\lambda \rho) d \lambda, \text { where } r=\sqrt{\rho^{2}+z^{2}}
$$

Then, we find that the value of the rapidly part in (36) is given by

$$
\int_{-\infty}^{\infty} h(\lambda, \rho) d \lambda=\int_{-\infty}^{\infty} \frac{\lambda}{u_{0}} e^{-u_{0}(m a+h)} H_{0}^{(2)}(\lambda \rho) d \lambda=\frac{2 e^{-i k_{0} r_{m}}}{r_{m}}
$$

While the slowly varying part in (36) is given by:

$$
f(\lambda) \approx \lambda^{2}\left(\frac{u_{12}^{-}}{u_{12}^{+}}\right)^{m} e^{-2 u_{1} m d}
$$

By replacing the slowly varying part with its value at the stationary phase point $\lambda=\lambda_{0}$, equation (38) becomes

$$
f\left(\lambda_{0}\right) \approx \frac{\rho^{2} k_{0}^{2}}{r_{m}^{2}}\left(\frac{1-\mathrm{n}}{1+\mathrm{n}}\right)^{m} e^{-2 i k_{1} m d}
$$

where $n=\frac{k_{2}}{k_{1}}$, and the approximation $u_{1} \approx i k_{1}$ is used because the stationary phase point ends up begin at $\lambda=k_{0}$.

Substituting from (37) and (39) in (36), we find that

$$
\Phi(m)=\frac{2 k_{0}^{2} \rho^{2}}{r_{m}^{3}}\left(\frac{1-n}{1+n}\right)^{m} e^{-i k_{0} r_{m}-2 \mathrm{i} k_{1} m d}
$$

Similarly by using the previous steps we get

$$
\begin{aligned}
& \Psi(m)=\frac{2 k_{0}^{2} \rho^{2}}{r_{m}^{3}}\left(\frac{1-n}{1+n}\right)^{m+1} e^{-i k_{0} r_{m}-2 \mathrm{i} k_{1}(m+1) d} \\
& \beta(m)=\frac{-2 k_{0}^{2} \rho^{2}}{r_{m+1}^{3}}\left(\frac{1-n}{1+n}\right)^{m} e^{-i k_{0} r_{m+1}-2 \mathrm{i} k_{1} m d} \\
& \gamma(m)=\frac{-2 k_{0}^{2} \rho^{2}}{r_{m+1}^{3}}\left(\frac{1-n}{1+n}\right)^{m+1} e^{-i k_{0} r_{m+1}-2 \mathrm{i} k_{1}(m+1) d}
\end{aligned}
$$

where

$$
r_{m+1}=\sqrt{\rho^{2}+((m+1) a+h)^{2}}
$$

Substituting from (40)-(43) into (36), finally yields the value of the vertical magnetic field $H_{z o}$ as follows

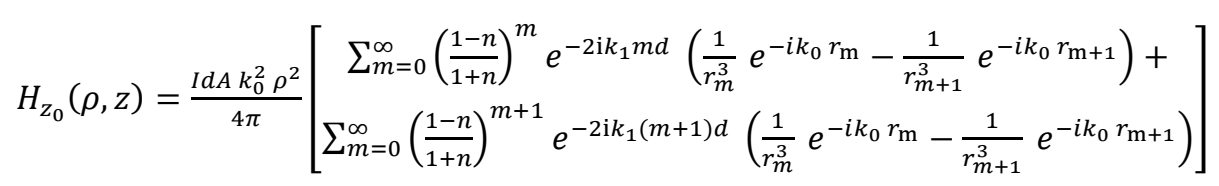

The physical meaning of (44) can be explained as follows: The first term represents a series of waves that propagate upward from the source and make m round trips, then travel along the surface in air and finally arrive at the 
field point in the air, where $\left(\frac{1-n}{1+n}\right)$ is the reflection coefficient at the surface of the lower half-space (bottom of the overburden slab). The reflection coefficient at the overburden surface is regarded as almost unity. The second term represents another series of waves, which propagate downward from the source first, then reflect upward at the surface, and travel along the same path as the former mentioned waves.

\section{Results and Discussions}

Using the simple technique [13] to evaluate $H_{\mathrm{zo}}$, we obtain a plot of vertical magnetic field versus the different values of frequencies. In these curves, we take; the vertical scale is the absolute value of the far-field of the vertical magnetic component $\left|H_{\mathrm{zo}}\right|$ versus the normalized frequencies. These plots are drawn with the normalized radial distance $[\mathrm{X}=$

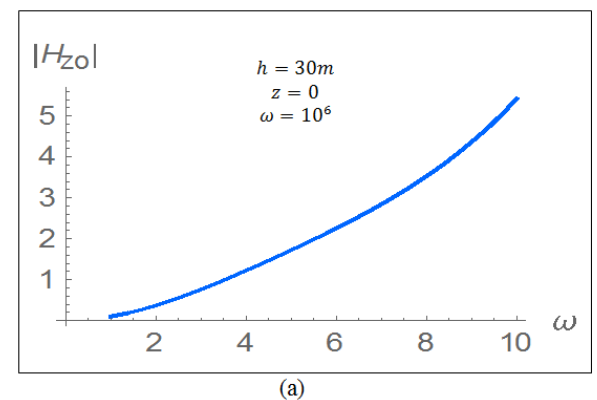

$\left.\rho \sigma_{1}\left(\frac{\mu_{0}}{\varepsilon_{1}}\right)^{\frac{1}{2}}=31.6\right]$ and the normalized overburden height $\left[\mathrm{D}=\mathrm{d} \sigma_{1}\left(\frac{\mu_{0}}{\varepsilon_{1}}\right)^{\frac{1}{2}}=3.16\right]$. The conductivities ratio is taken as $\left[\left(\frac{\sigma_{2}}{\sigma_{1}}\right)=100\right],\left[\sigma_{1}=10^{-3}\right]$, while the permittivities are $\left[\varepsilon_{1}=10 \varepsilon_{0}\right],\left[\varepsilon_{2}=100 \varepsilon_{0}\right]$, and the source height $[h=30 \mathrm{~m}]$. In these figures, the vertical scale is normalized by the factor $\left(\frac{I d A \rho^{2}}{4 \pi}\right)$ to show the absolute value of the farfield of the vertical magnetic component versus the normalized frequencies. To attain the numerical calculation, we ascertain that $\left(\sum_{m=0}^{5}\right)$ is a match for $\left(\sum_{m=0}^{\infty}\right)$ under the conditions shown in Figures. Therefore, we adopt the $5^{\text {th }}$ partial sum (i.e. $\mathrm{m}=0,1,2----5$ ) instead of the infinite one. We observe from these plots that increasing the frequency increases the waveguide mode contribution. These plots coincide with previous results obtained using other methods.

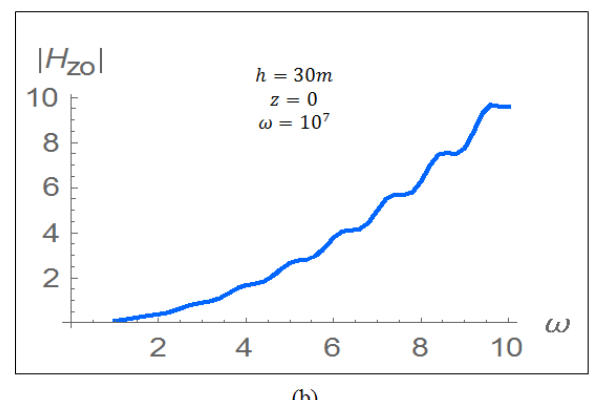

(b)

Figure 2. Variation of the vertical magnetic field $\left|H_{z o}\right|$ in frequency-domain in the air region at $h=30, \mathrm{mz}^{2}=0$, and different values of $\omega=10^{6}, 10^{7}$.
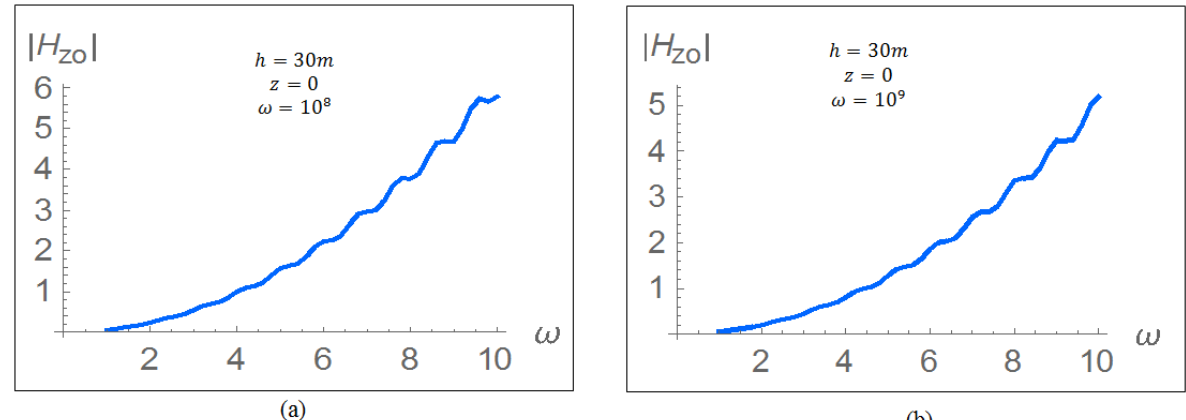

(b)

Figure 3. Variation of the vertical magnetic field $\left|H_{z o}\right|$ in frequency-domain in the air region at $h=30 \mathrm{~m}, z=0$, and different values of $\omega=10^{8}, 10^{9}$.

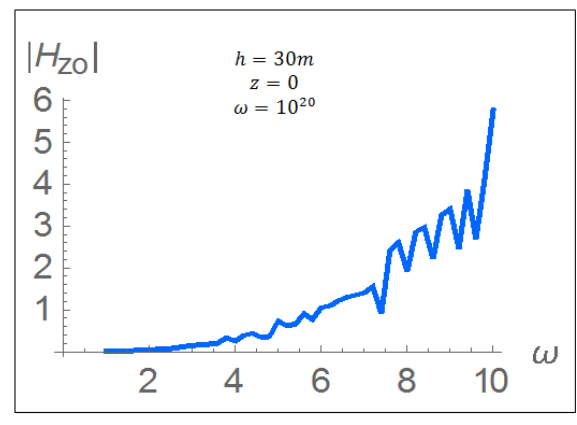

(a)

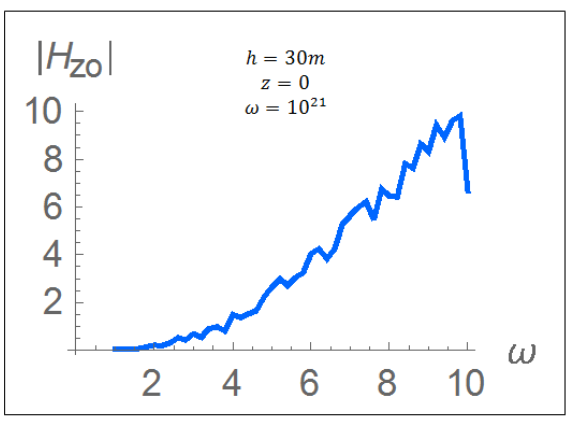

(b)

Figure 4. Variation of the vertical magnetic field $\left|H_{z o}\right|$ in frequency - domain in the air region at $h=30 \mathrm{~m}, z=0$, and different values of $\omega=$ $10^{20}, 10^{21}$. 


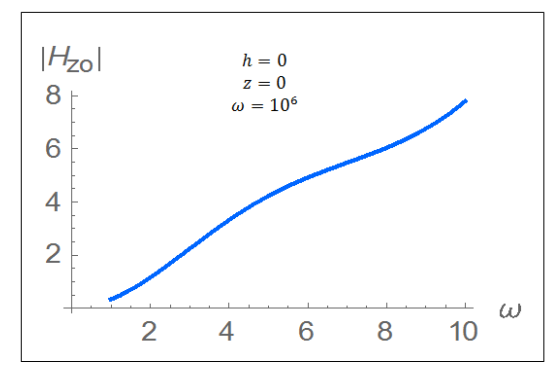

(a)

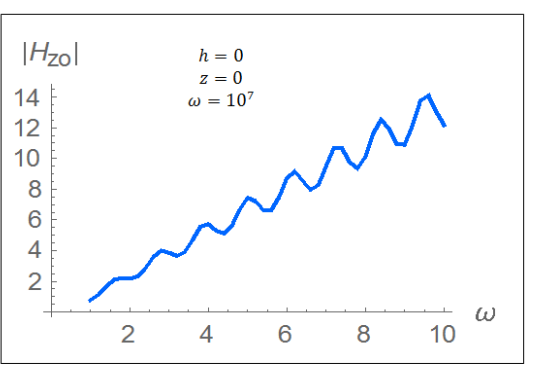

(b)

Figure 5. Variation of the vertical magnetic field $\left|H_{z o}\right|$ in frequency-domain in the air region at $h=0 \mathrm{~m}, z=0$, and different values of $\omega=10^{6}, 10^{7}$.
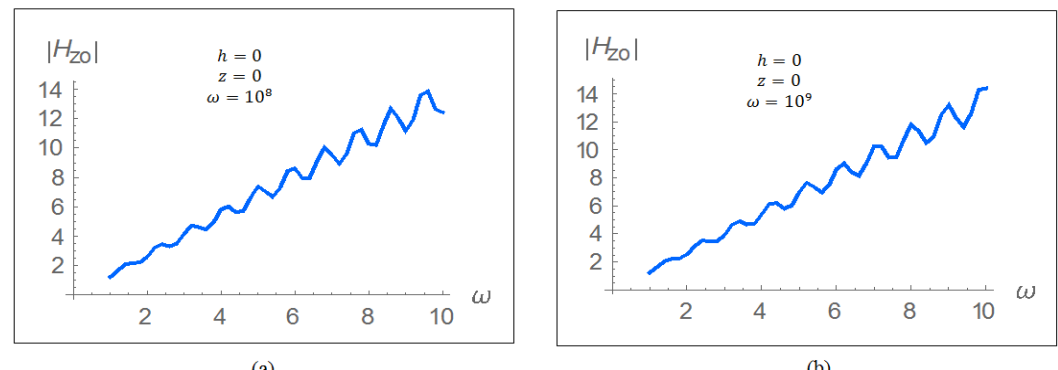

Figure 6. Variation of the vertical magnetic field $\left|H_{z o}\right|$ in frequency-domain in the air region at $h=0 \mathrm{~m}, z=0$, and different values of $\omega=10^{8}, 10^{9}$.

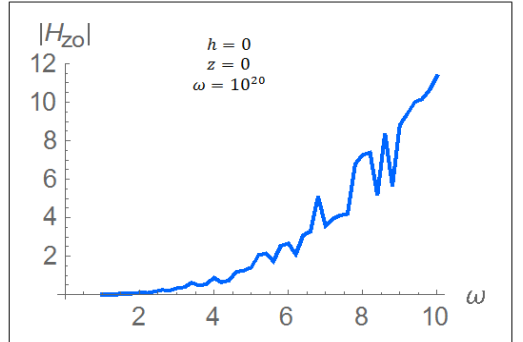

(a)

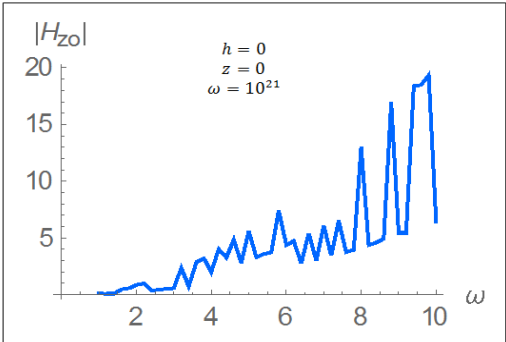

(b)

Figure 7. Variation of the vertical magnetic field $\left|H_{z o}\right|$ in frequency-domain in the air region at $h=0 \mathrm{~m}, z=0$, and different values of $\omega=10^{20}, 10^{21}$.

\section{Conclusion}

The main purpose of this is to derive Closed-form formulae for the far-field excited by the loop source located at a height $(\mathrm{h})$ in the air layer of a planar two-layered ground rapidly and simply. We apply the technique [13] to evaluate Sommerfeld integral with the help of the complex image theory [14]. The method hinges on the use of some integral identities and the identification of the stationary phase point of the integrand. The location of the stationary phase point is easily remembered from its physical interpretation. The result using this method always agree to the leading order with the result using conventional stationary phase method or saddlepoint method. This method is simple, effective and much quicker than the rather long methods, it can be also easily used to get the far-field formulas for other similar problems.

\section{References}

[1] R. K. Moore and W. E. Blair, "Dipole radiation in a conducting half space," Journal of Research of the National Bureau of Standards Section D: Radio Propagation, vol. 65, no. 6 , pp. 547-563, 1961.
[2] A. Banos, "Dipole Radiation in the Presence of a Conducting Half-Space," Pergamon Press, Oxford, NY, USA, 1966.

[3] J. R. Wait, "The electromagnetic fields of a horizontal dipole in the presence of a conducting half-space," Can. J. Phys., vol. 39, pp. 1017-1028, 1961.

[4] J. A. Kong, "Electromagnetic Wave theory," John Wiley \& Sons, New York, NY, USA, 1986.

[5] J. R. Wait, "Electromagnetic waves in stratified media," Pergamon Press, New York, NY, USA, 1970.

[6] W. C. Chew, J. A. Kong, "Electromagnetic field of a dipole on a two layer earth", Geophys., vol. 46, pp. 309-315, 1981.

[7] S. H. Ward and G. W. Hohmann, "Electromagnetic theory for geophysical applications," in Electromagnetic Methods in Applied Geophysics, vol. 1, chapter 4, pp. 130-311, Society of Exploration Geophysicists, Tulsa, Okla, USA, 1988.

[8] R. E. Colline, "Antennas and Radio Wave Propagation," New York, McGraw-Hill, 1985.

[9] Y. Long, H. Jiang and Y. Lin "Electromagnetic field due to loop antenna in a borehole," IEEE Trans. Geosci. Remote Sensing, vol. 34, pp. 33-35, Jan. 1996. 
[10] A. Arutaki and J. Chiba, "Communication in a three-layered conducting media with a vertical magnetic dipole," IEEE Trans. Antennas Propagat., vol. 28, pp. 551-556, July 1980.

[11] A. Sommerfeld, "Partial differential equations in physics," Academic Press. New York and London, 1964.

[12] W. C. Chew, "A quick way to approximate a SommerfeldWely-Type integral,” IEEE. Trans. Antennas Propagat., vol. 36, no. 11, pp. 1654-1657, Nov. 1988.

[13] Y. Long, H. Jiang and B. Rembold, "Far-Region Electromagnetic Radiation with a Vertical Magnetic Dipole in Sea," IEEE Trans. Antennas Propagat., vol. 49, no. 6, pp. 992996, June 2001.

[14] P. R. Bannister, "The image theory of electromagnetic fields of a horizontal electric dipole in the presence of conducting half-space," Radio Sci., vol. 17, no. 5, pp. 1095-1102, Sept.Oct. 1982.
[15] M. Parise, M. Muzi, and G. Antonini, "Loop Antennas with Uniform Current in Close Proximity to the Earth: Canonical Solution to the Surface-To-Surface Propagation Problem," Progress In Electromagnetics Research B, vol. 77, pp. 57-69, 2017.

[16] J. A. Stratton, "Electromagnetic Theory," New York, McGraw-Hill, 1941.

[17] D. S. Jones, "The Theory of Electromagnetism," London, U. K.: Pergamon, 1964.

[18] M. Abramowitz and I. A. Stegun, "Handbook of Mathematical Functions,” New York: Dover, 1972. 